\title{
Taking a bite out of hypertrophic cardiomyopathy: soy diet and disease
}

\author{
Cathy J. Hatcher and Craig T. Basson \\ Molecular Cardiology Laboratory, Greenberg Division of Cardiology, Department of Medicine, \\ Weill Medical College of Cornell University, New York, New York, USA.
}

\begin{abstract}
Some forms of hypertrophic cardiomyopathy (HCM) are caused by mutations in cardiac sarcomeric genes, but environmental factors are believed to influence the hypertrophic response. A highly variable but potentially significant environmental factor is diet. Since soy-rich diets have been speculated to confer protection against cardiovascular disease, Stauffer et al. have explored the influence of a soy diet on cardiac growth and function in a transgenic mouse model of HCM (see the related article beginning on page 209). They report that mice fed a soy diet exhibited significantly worse HCM than mice fed a soy-free (milk protein) diet. This study provides the first evidence of an environmental modifier diet - on the hypertrophic phenotype and has implications for the way in which disease phenotypes are assessed in genetically altered murine models of disease.
\end{abstract}

Genetically altered mouse models are commonly used to study human diseases. However, little attention has been paid to the potential influence of diet on disease phenotype. In this issue of the JCI, Stauffer, Leinwand, and colleagues report a dramatic adverse effect of a soy-based diet on the severity of a cardiac phenotype in mice (1). Male mice expressing a mutant isoform of $\alpha$-myosin heavy chain that is linked to hypertrophic cardiomyopathy (HCM) in humans were fed a soy-based diet. These mice exhibited significantly worse heart disease compared with comparable animals fed a milk protein-based (casein) diet (Figure 1). This study has implications for both diet-related response to heart disease and the assessment of disease phenotypes in murine models.

\section{Hypertrophic cardiomyopathy}

$\mathrm{HCM}$ is a common genetic cardiovascular disease occurring in 1 of 500 individuals (2). This Mendelian autosomal dominant disease is characterized by a hypertrophied $\mathrm{LV}$ in the absence of another cardiac or systemic disease capable of producing this

Nonstandard abbreviations used: HCM, hypertrophic cardiomyopathy.

Conflict of interest: The authors have declared that no conflict of interest exists.

Citation for this article: J. Clin. Invest. 116:16-19 (2006). doi:10.1172/JCI27455. magnitude of hypertrophy. LV hypertrophy may be symmetric or asymmetric. The symmetric form is characterized by mild, concentric thickening of the LV with a reduced ventricular cavity (3). However, hypertrophy is more commonly asymmetric with disproportionate thickening of the interventricular septum (3).

Along with a hypercontractile LV, features of HCM may include dynamic subaortic obstruction and systolic anterior motion of the mitral valve leaflets that can combine to produce obstructive physiology and a systolic murmur. Murmur intensity varies with physical examination and/or medications that alter ventricular loading. Regardless of the presence or absence of subaortic obstruction, individuals can present with shortness of breath and chest discomfort resulting from diastolic dysfunction and/or subendocardial ischemia in the hypertrophied ventricle. Palpitations may herald life-threatening atrial and ventricular tachyarrhythmia whose incidence is often unrelated to the severity of ventricular hypertrophy. Of HCM patients, $5-10 \%$ will progress from a compensated to a decompensated state in which the hypertrophied LV begins to dilate, ventricular walls thin, and irreversible heart failure due to systolic dysfunction ensues. Such a dilated phase represents a late stage of the disease with poor prognosis.

Cardinal histological features of HCM include myocyte hypertrophy, myocyte disarray, and interstitial fibrosis (4). Disarray may be focal or widely distributed throughout the LV wall and tends to be more extensive in younger patients who succumb to the disease (2). Another histopathological feature of HCM is small vessel disease, or arterial dysplasia, characterized by narrowing of the intramural coronary arteries secondary to wall thickening from increased intimal and medial collagen deposition. These microvascular changes likely contribute to impaired coronary vasodilator reserve and myocardial ischemia. Consequent myocyte death leads to myocardial scarring and fibrosis that may complicate transition from the compensated HCM phase to the decompensated, dilated phase.

HCM is often referred to as a cardiac sarcomere disease because it can be caused by mutations in genes encoding thick and thin filament sarcomeric proteins involved in contractility. Most HCM-causing mutations occur in 3 genes, namely those encoding $\beta$-myosin heavy chain, cardiac troponin $\mathrm{T}$, and myosin-binding protein $\mathrm{C}(5-7)$. The remainder of the HCM-related mutant genes includes those encoding $\alpha$-tropomyosin, cardiac troponin I, regulatory and essential myosin light chains, titin, troponin $C, \alpha$-actin, and $\alpha$-myosin heavy chain (8). Although mutation hot spots exist, most unrelated individuals possess unique mutations. Correlations have been proposed between disease severity/ prognosis and the different genes as well as specific mutations in any given gene. For example, some $\beta$-myosin heavy chain mutations cause a severe phenotype with early onset and increased risk of sudden cardiac death. However, there are marked intrafamilial variations in HCM phenotypes that suggest epigenetic and environmental factors can modify the cardiac hypertrophic response and disease prognosis. Although there are many environmental factors influencing lifestyle, one key factor is diet. 


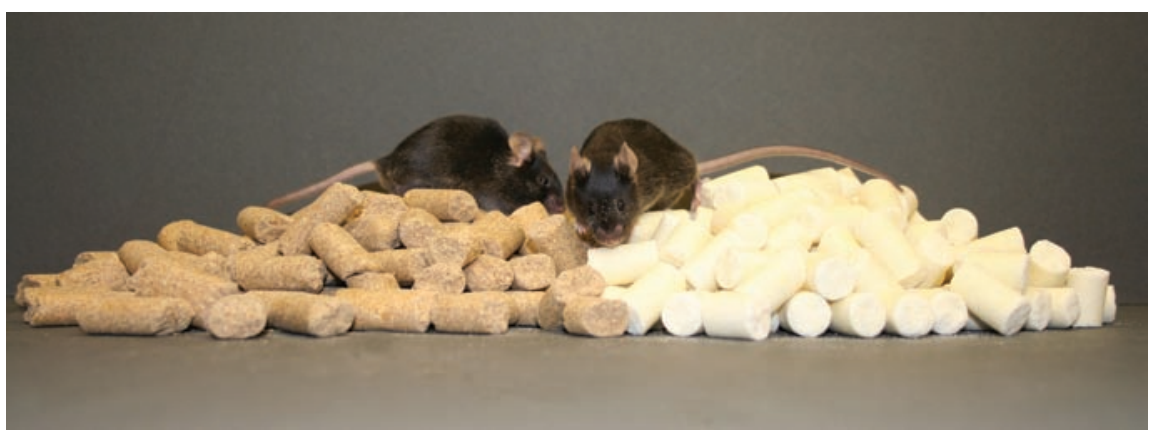

Figure 1

HCM mice fed a phytoestrogen-supplemented soy diet versus a soy-free casein diet. Significant changes in cardiac growth were observed in male HCM mice fed a phytoestrogen-supplemented soy diet (left) in comparison with mice fed a soy-free casein diet (right) (1). Photograph courtesy of Leslie A. Leinwand, University of Colorado.

\section{Impact of phytoestrogens on health}

For years, soy-rich diets have been speculated to possess beneficial physiologic effects for general health, particularly in the cardiovascular system. Soy intake in the average Southeast Asian diet is estimated at $20-50 \mathrm{mg} / \mathrm{d}$. Increased awareness of the beneficial effects of soy proteins has contributed to its rapid growth in inclusion in Western diets, with soy intakes ranging from $0.15-3 \mathrm{mg} / \mathrm{d}$ in the United States (9). This has helped create a billion dollar industry for soy foods and dietary supplements in the United States alone. Beneficial as well as adverse effects of soy have been proposed in clinical and laboratory settings. Cancer prevention and cholesterol-lowering properties of soy proteins are among the proposed benefits whereas increased androgen levels and decreases in thyroid peroxidase levels are among the known adverse effects (10).

Biological consequences of soy-rich diets have been attributed to the presence of soy isoflavones, which are members of the phytoestrogen family. Phytoestrogens are plant-derived, estrogen-like compounds capable of interacting with the estrogen receptors $E R \alpha$ and $E R \beta$ because their structure resembles that of the endogenous hormone $17 \beta$-estradiol (11). Four groups of phenolic compounds are classified as phytoestrogens: isoflavones, stilbenes, coumestans, and lignans. The main isoflavones, found mostly in soybeans, are genistein, daidzein, and glycitein (11). Several epidemiological studies (12-14) have concluded that phytoestrogens confer cardioprotection by downregulating cardiovascular disease risk markers. Soy product consumption has been associated with the following: (a) reduced serum cho- lesterol levels via reductions in total and low-density lipoprotein levels; (b) vasodilatory activity under certain conditions; and (c) enhanced activity of certain antioxidant enzymes to increase antioxidant activities. Most of these studies demonstrated consistent improvement in endothelial function while others were unable to demonstrate improvements in serum lipid levels and antioxidant activity (12-14).

\section{Influence of soy diet on heart disease}

In this issue of the JCI, Stauffer et al. (1) describe the influence of a dietary modification on improvement of disease indicators and cardiac function in a transgenic mouse model of HCM. They report further study of a transgenic mouse model endowed with cardiac overexpression of the $\alpha$-myosin heavy chain mutant isoform. Unlike humans, in which the $\beta$-myosin heavy chain is the predominant adult cardiac myosin isoform, $\alpha$-myosin heavy chain is predominant in mice. In these transgenic mice, created by Leinwand and colleagues (15), the overexpressed $\alpha$-myosin heavy chain gene carries an R403Q missense mutation (that also causes human HCM) as well as a 59-base pair deletion. The resulting phenotype is sex-dependent cardiomyopathy. Female mice develop HCM while male mice exhibit thinning of the ventricular walls and systolic dysfunction reminiscent of dilated HCM. Sex differences in HCM manifestations have been seen in other HCM models (16).

In this HCM model, Stauffer et al. (1) now observe a significant increase in relative cardiac growth in male HCM mice consuming a phytoestrogen-supplemented diet in comparison with female HCM mice as well as male HCM mice fed a soy-free diet. They suggest that the diet supplemented with phytoestrogens, major estrogen receptor ligands, contributes to the cardiac growth differences observed between the sexes via a sex-steroid receptor-mediated mechanism. Previous investigations have described an influence of sex steroids on cardiac growth (17). However, sex-dependent alterations on myocardial estrogen receptors have not been described. The authors hypothesize that female animals, who are constantly exposed to endogenous circulating levels of estrogen compounds, are less sensitive to augmented estrogenic exposures than males (Figure 2). Therefore, the effective estrogen dose may be the key for eliciting cardiac growth and, hence, a hypertrophic response.

The authors noted a marked improvement of hypertrophic progression in this mouse HCM model when the animals were switched to a soy-free diet (1). Under the influence of a soy-free diet, mice no longer transitioned from a compensated phase to decompensated, dilated HCM. Stauffer and colleagues sought to reproduce their soy-diet findings by providing for these mice a phytoestrogen-supplemented diet containing the 2 predominant isoflavones, genistein and daidzein. However, supplementation with these isoflavones insufficiently reproduced the murine phenotype observed with the soy diet. This finding suggests potential significance of the third, less prevalent, isoflavone, glycitein, and its cooperativity with genistein and daidzein in producing depressed ventricular contractility accompanied by wall thinning and LV chamber dilation.

With increasing attention to the role of soy proteins in cardiovascular health, these new data provide a strong link between soy diet and progression of cardiac disease. Stauffer et al. (1) consider a molecular mechanism whereby soy proteins might mediate these deleterious effects through induction of augmented cell growth and apoptosis (Figure 2). A similar mechanism has been suggested in cardiac fibroblasts (17). Hence, this discovery may lead to dietary modifications preventing formation or progression of some hypertrophic cardiac diseases. Scientists have previously suggested that lifestyle factors influence phenotypic variability to the hypertrophic response. Stauffer et al. (1) are the first to provide clear evidence of a discrete environmental modifier - dietary content - on the hypertrophic phenotype. 
A
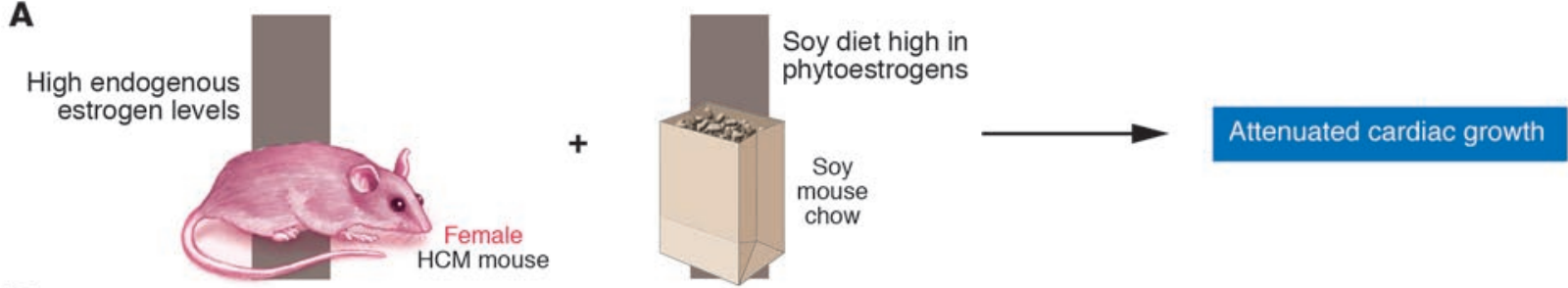

B
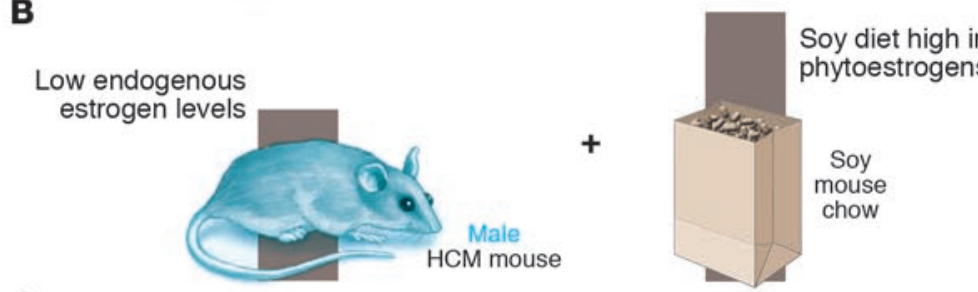

C
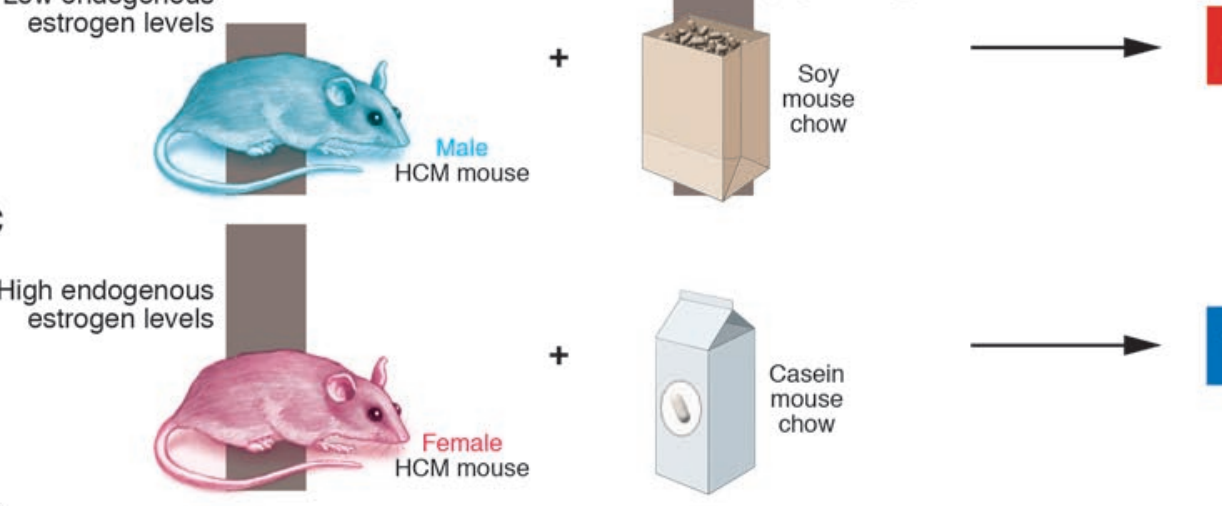

Augmented cardiac growth

Heart failure

Potential underlying mechanisms? Increased IGF-1/Akt/GSK3 $\beta$ activity

Increased $\beta$-myosin heavy chain synthesis Increased activation of caspase-3 Increased cardiomyocyte apoptosis Sex steroid receptor-mediated

D
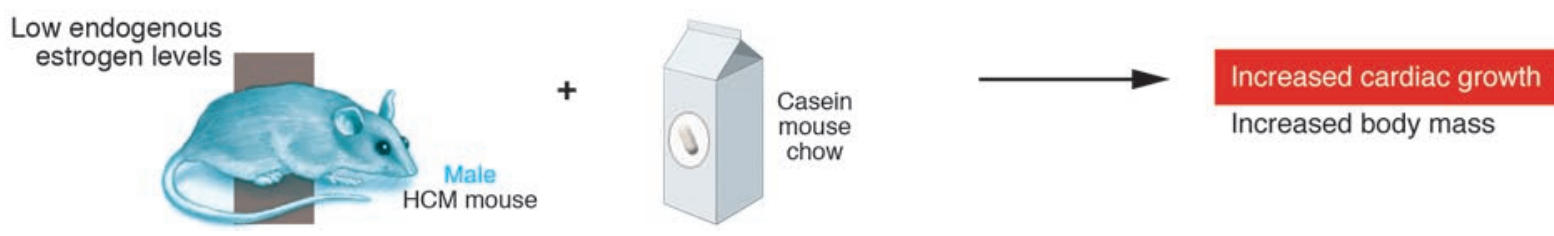

Figure 2

Soy diet alters the physiological cardiac response to HCM in a sex-dependent manner (1). (A) Female HCM mice, which have high endogenous levels of estrogen, were placed on a standard soy diet high in phytoestrogens. Cardiac growth was attenuated in these mice, and contractile function was preserved. (B) Male HCM mice, who have low endogenous levels of estrogen, consumed a standard soy diet high in phytoestrogens. Relative cardiac growth was augmented in these mice. A decrease in contractile function was also observed along with LV chamber dilation and wall thinning. The potential molecular and physiologic mechanisms underlying these events are shown. All HCM mice consuming a soy-free, casein diet were significantly heavier than those consuming the standard soy diet. However, the female HCM mice consuming a soy-free, casein diet (C) did not demonstrate a hypertrophic response whereas the male HCM mice did (D). In addition, the casein diet did not negatively impact contractile function in these mice.

\section{Future implications of dietary influences on animal model phenotypes}

From a broader perspective, the findings by Stauffer et al. (1) may influence the decisions research investigators make with regard to dietary considerations for a wide variety of animal models used for studying human disease. Individual researchers and institutional animal health facilities strive to provide their animals with high-quality feed essential for meeting their physiological requirements for growth and reproduction. Numerous laboratory diet products are on the market. Their nutritional content varies among formulations as well as among manufacturers. Most natural- ingredient diets have a "closed" formula because individual components of the diet are not specified. Use of these diets is risky because the ingredients change in association with commodity prices. The less commonly used "open" formula diets are manufactured in accordance with an established known ingredient formulation. As the controversial role of dietary phytoestrogens is deciphered and appreciated, feed manufacturers have begun to produce diets lacking protein sources known to contain isoflavones (18). Previous data have demonstrated the influence of diet, both positive and negative, on the progression of certain diseases indigenous to specific rodent strains, e.g., chronic nephropathy in Fischer 344 rats $(19,20)$.
Taken together with the current report, researchers should conscientiously consider the diet consumed by their animals and how it may influence the manifestation of disease phenotypes within various genetic backgrounds.

\section{Acknowledgments}

C.J. Hatcher is supported by a grant from the NIH National Heart, Lung, and Blood Institute (K01HL080948). C.T. Basson is an Established Investigator of the American Heart Association and an Irma T. Hirschl Scholar. The authors are grateful to Neil S. Lipman for comments.

Address correspondence to: Craig T. Basson, Cardiovascular Research, Greenberg 
Division of Cardiology, Department of Medicine, Weill Medical College of Cornell University, 525 East 68th Street, New York, New York 10021, USA. Phone: (212) 746-2201; Fax: (212) 746-2222; E-mail: ctbasson@med.cornell.edu.

1. Stauffer, B.L., Konhilas, J.P., Luczak, E.D., and Leinwand, L.A. 2006. Soy diet worsens heart disease in mice. J. Clin. Invest. 116:209-216. doi:10.1172/ JCI24676.

2. Maron, B.J., et al. 1995. Prevalence of hypertrophic cardiomyopathy in a general population of young adults. Echocardiographic analysis of 4111 subjects in the CARDIA Study. Coronary Artery Risk Development in (Young) Adults. Circulation. 92:785-789.

3. Davies, M.J., and McKenna, W.J. 1995. Hypertrophic cardiomyopathy-pathology and pathogenesis. Histopathology. 26:493-500.

4. Klues, H.G., Schiffers, A., and Maron, B.J. 1995. Phenotypic spectrum and patterns of left ventricular hypertrophy in hypertrophic cardiomyopathy: morphologic observations and significance as assessed by two-dimensional echocardiography in 600 patients. J. Am. Coll. Cardiol. 26:1699-1708.

5. Maron, B.J. 2002. Hypertrophic cardiomyopathy: a systematic review. J. Am. Med. Assoc. 287:1308-1320.
6. Maron, B.J., et al. 2003. American College of Cardiology/European Society of Cardiology clinical expert consensus document on hypertrophic cardiomyopathy. A report of the American College of Cardiology Foundation Task Force on Clinical Expert Consensus Documents and the European Society of Cardiology Committee for Practice Guidelines. J. Am. Coll. Cardiol. 42:1687-1713.

7. Spirito, P., Seidman, C.E., McKenna, W.J., and Maron, B.J. 1997. The management of hypertrophic cardiomyopathy. N. Engl. J. Med. 336:775-785.

8. Ahmad, F., Seidman, J.G., and Seidman, C.E. 2005 The genetic basis for cardiac remodeling. Annu. Rev. Genomics Hum. Genet. 6:185-216.

9. Horn-Ross, P.L., et al. 2001. Phytoestrogen consumption and breast cancer risk in a multiethnic population: the Bay Area Breast Cancer Study. Am. J. Epidemiol. 154:434-441.

10. Sirtori, C.R., Arnoldi, A., and Johnson, S.K. 2005. Phytoestrogens: end of a tale? Ann. Med. 37:423-438.

11. Cornwell, T., Cohick, W., and Raskin, I. 2004. Dietary phytoestrogens and health. Phytochemistry. 65:995-1016.

12. Squadrito, F., et al. 2002. The effect of the phytoestrogen genistein on plasma nitric oxide concentrations, endothelin-1 levels and endothelium dependent vasodilation in postmenopausal women. Atherosclerosis. 163:339-347.

13. Vega-Lopez, S., et al. 2005. Plasma antioxidant capacity in response to diets high in soy or animal protein with or without isoflavones. Am. J. Clin. Nutr. 81:43-49.

14. Dewell, A., Hollenbeck, C.B., and Bruce, B. 2002. The effects of soy-derived phytoestrogens on serum lipids and lipoproteins in moderately hypercholesterolemic postmenopausal women. J. Clin. Endocrinol. Metab. 87:118-121.

15. Vikstrom, K.L., Factor, S.M., and Leinwand, L.A. 1996. Mice expressing mutant myosin heavy chains are a model for familial hypertrophic cardiomyopathy. Mol. Med. 2:556-567.

16. Maass, A.H., Ikeda, K., Oberdorf-Maass, S., Maier, S.K., and Leinwand, L.A. 2004. Hypertrophy, fibrosis, and sudden cardiac death in response to pathological stimuli in mice with mutations in cardiac troponin T. Circulation. 110:2102-2109.

17. Dubey, R.K., Gillespie, D.G., Jackson, E.K., and Keller, P.J. 1998. 17Beta-estradiol, its metabolites, and progesterone inhibit cardiac fibroblast growth. Hypertension. 31:522-528.

18. Thigpen, J.E., et al. 1999. Phytoestrogen content of purified, open- and closed-formula laboratory animal diets. Lab. Anim. Sci. 49:530-536.

19. Iwasaki, K., et al. 1988. The influence of dietary protein source on longevity and age-related disease processes of Fischer rats. J. Gerontol. 43:B5-B12.

20. Shimokawa, I., et al. 1993. Diet and the suitability of the male Fischer 344 rat as a model for aging research. J. Gerontol. 48:B27-B32.

\title{
Gene expression profiling gets to the root of human hair follicle stem cells
}

\author{
George Cotsarelis
}

University of Pennsylvania School of Medicine, Philadelphia, Pennsylvania, USA.

\begin{abstract}
Hair follicle stem cells sustain growth and cycling of the hair follicle and are located in the permanent portion of the follicle known as the bulge. In this issue of the JCI, Ohyama et al. report the characterization of global gene expression patterns of human hair follicle stem cells after their isolation using sophisticated laser capture techniques to microdissect out bulge cells (see the related article beginning on page 249). They discovered a panel of cell surface markers useful for isolating living hair follicle stem cells, a finding with potential therapeutic implications since isolated stem cells in mice can generate new hair follicles when transplanted to other mice. The findings of Ohyama et al. validate the use of the mouse for studying hair follicle biology but also underscore critical differences between mouse and human stem cell markers. In particular, CD34, which delineates hair follicle stem cells in the mouse, is not expressed by human hair follicle stem cells, while CD200 is expressed by stem cells in both species. Ultimately, this information will assist efforts to develop cell-based and cell-targeted treatments for skin disease.
\end{abstract}

Advances in stem cell biology have resulted in major clinical benefits. Bone marrow transplantation for treatment

Nonstandard abbreviations used: K15, cytokeratin 15; LRC, label-retaining cell.

Conflict of interest: The author has declared that no conflict of interest exists.

Citation for this article: J. Clin. Invest. 116:19-22 (2006). doi:10.1172/JCI27490. of cancers and corneal transplantation for treatment of blindness resulting from chemical burns were both possible because stem cells were identified and isolated from the affected tissues $(1,2)$. Many disorders of the skin, such as cancer, chronic wounds, skin atrophy and fragility, hirsutism, and alopecia, can be viewed as disorders of adult stem cells. Because stem cells in the epidermis and hair follicle serve as the ultimate source of cells for both of these tissues, understanding the control of their proliferation and differentiation is key to understanding disorders related to disruption in these processes. Furthermore, the isolation, cultivation, and propagation of epithelial stem cells are important for tissue-engineering approaches to treating skin disorders (3).

The skin possesses an outer covering produced by the epidermis that protects us from dehydration and from external environmental insults. The outer layer of the hair follicle is contiguous with the epidermis, forming invaginations that, in the case of scalp follicles, penetrate deeply into the fat underlying the skin. Approximately 5 million follicles that are spread over the body generate hair in a cyclical fashion. The duration of anagen (the period of hair growth), which varies from less than 60 to more than several thousand days on different body sites, determines the length of the hair shaft. After anagen, the follicle enters a stage of involution (catagen) and then a stage of 